\title{
Translating Subjective Data to Objective Measures to Drive Product Design and Experience
}

\author{
Erin K. Walline and Bradley Lawrence \\ Dell, Inc., Experience Design Group, \\ One Dell Way, Round Rock, Texas, 78682 \\ \{Erin_Walline, Bradley_Lawrence\}@Dell.com
}

\begin{abstract}
To successfully drive best-in-class human factors into product design, it is sometimes necessary to adopt more non-traditional experimental methods and reporting techniques. Within the PC industry, a traditional usability study is usually comprised of running eight to twelve participants through a set of tasks in a two-hour time period, collecting and reporting ease-of-use, success rate, time-on-task, and preference data. This traditional method is great at identifying potential usability pitfalls, but not necessarily equipped to focus on a product's visual appeal or quality perception. Two case studies are described that introduce non-traditional methods which: (1) focus on the perceived quality of specific product designs; (2) relate subjective data to concrete mechanical terms such that engineers have clear direction on how to build the products; and (3) report findings in a concise, graphical manner that is easily and quickly understood by executives and colleague functions lacking a human factors background.
\end{abstract}

Keywords: Product design, experience, usability.

\section{Case Study I: Hard Drive Removal Force}

\subsection{Introduction}

This research effort came from the side of the corporate organization where IT customers purchasing enterprise-level servers and storage devices were complaining about the feel and forces necessary to remove and replace front-access hard drives from these systems. Hard drives comprise the heart of an enterprise's storage and data and customer council feedback was indicating the perceived quality associated with these replacements was not instilling trust in the overall product line. While failure rates are very low on individual hard drives, when a data center houses over thousands, removals and replacements can occur as frequently as weekly, exacerbating the exposure to the perceived quality issue.

\subsection{Method}

Twenty IT professionals were recruited to participate in an investigation study where the entire study involved multiple removals and replacements of hard drives across 
several manufacturers and several product generations (depicted in Figure 1). The smoothness (defined as change in force per unit of travel) and peak force varied widely among the multiple systems tested, and there was enough resolution such that the IT professionals could detect subtle to great differences among the various designs. IT customers rated how "right" the forces and smoothness felt on a 7-point scale from "too much" to "just right" (the midpoint) to "too little." In parallel, the actual smoothness and force data were collected quantitatively.

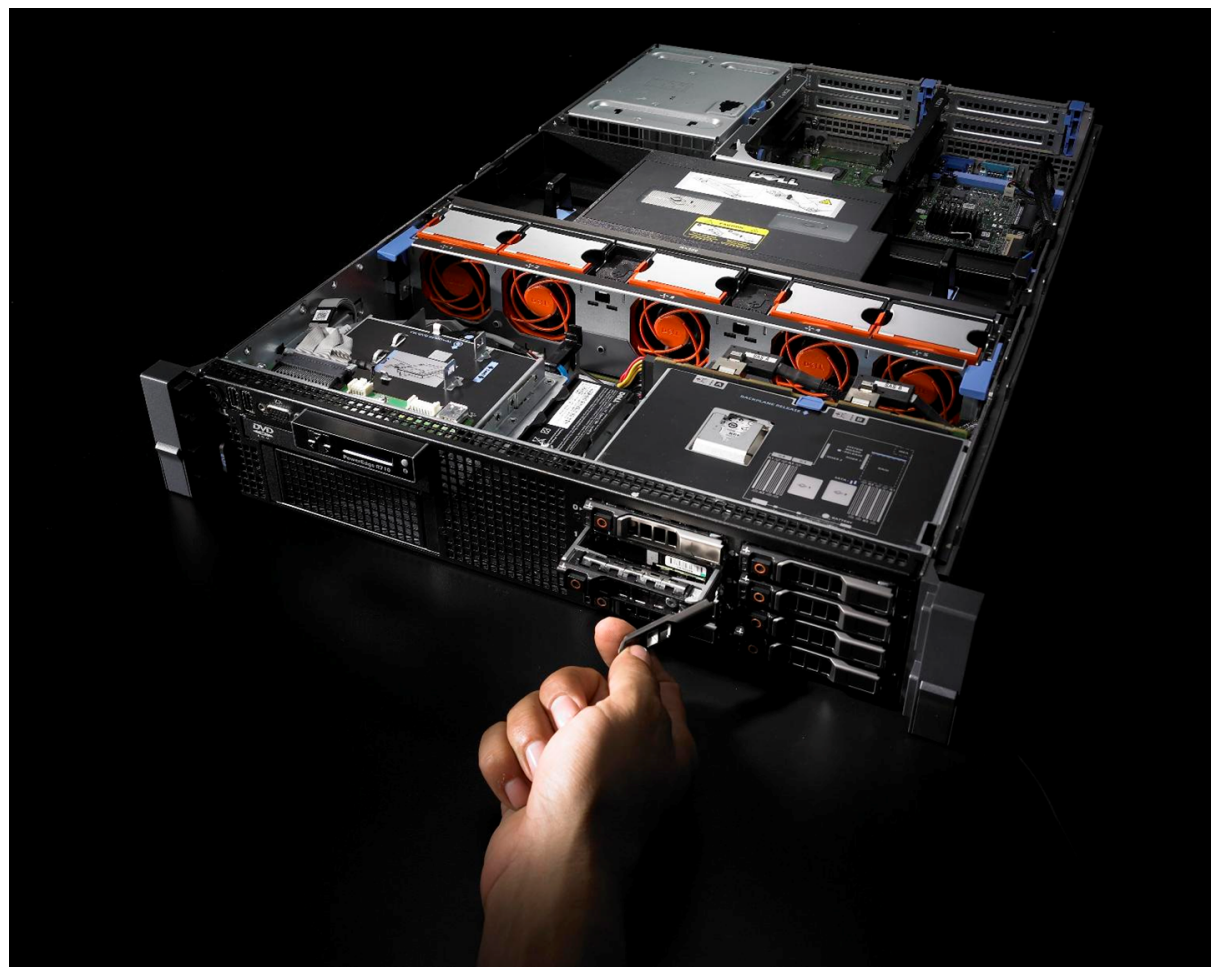

Fig. 1. Hard drive removal from a server

\subsection{Results}

Figure 2 shows the averages of the twenty participants' ratings of perceived smoothness and force for three representative hard drives of the multiple hard drives tested. Figure 3 shows the actual force profiles that were measured using a fixture and force gauge at every $10 \mathrm{~mm}$ of travel along the length of the hard drive removal for these three representative samples.

Figures 2 and 3 indicate thresholds for both smoothness and force acceptability. Figure 2 shows perceived smoothness is only acceptable for hard drive \#3 (HDD 3), indicated by the bracket on Figure 3 that is just less than one unit of force across the range of travel. Figure 2 shows however that both HDD 2 and HDD 3 are in the acceptable range corresponding to two units of force (the dotted line in Figure 3). 


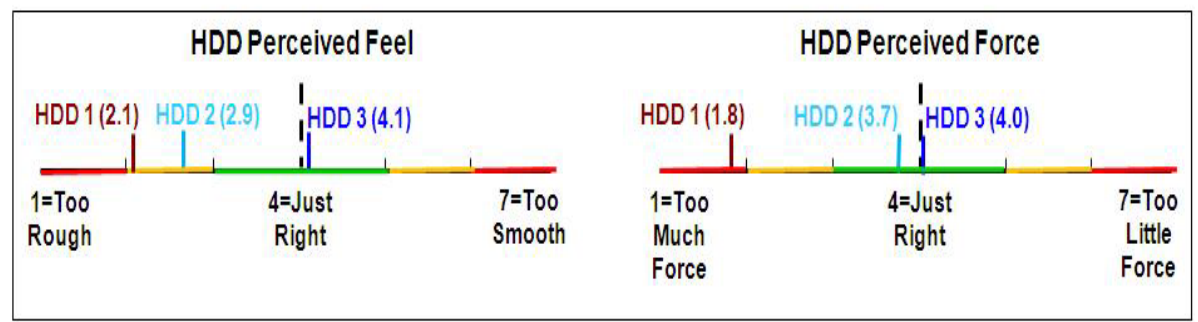

Fig. 2. Perceived hard drive removal smoothness and force

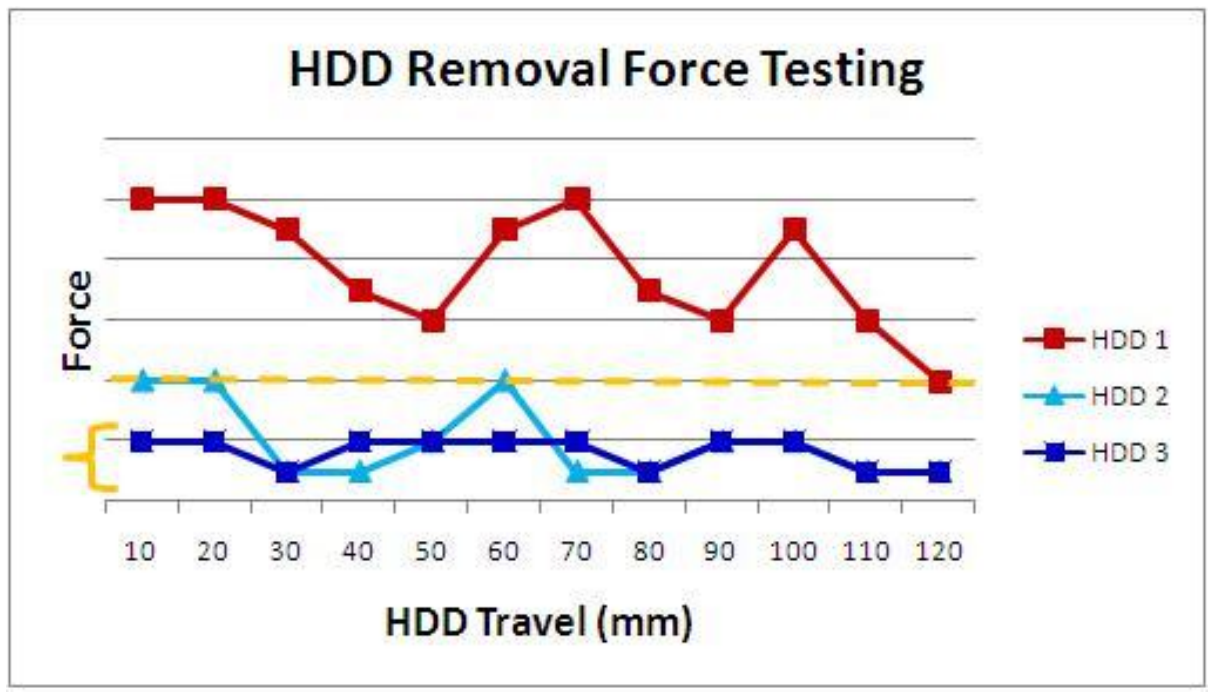

Fig. 3. Actual measured hard drive removal force profiles

When both data sources were presented together graphically in one easily digestible PowerPoint slide, the reporting technique became known across the organization as "Goldilocks" analysis and was received in a very positive light providing very clear, comprehensible direction for building the best hard drive carrier. Executives and peers across organizations have come to anticipate this type of simplified methodology and reporting in future research of this kind. Further, "Goldilocks" testing targeted nearly every subsystem and component on the server product line for hard drive carrier improvement. Perceived quality improvements were made across the board, and customer feedback from customer councils, reviews, sales calls, etc. indicate that these servers are now seen as serious enterprise hardware.

\section{Case Study II: “Plasticky" Perception}

\subsection{Introduction}

After receiving direct customer feedback, as well as media reviews, that identified products as feeling too cheap and "plasticky," an investigation was undertaken to 
determine what factors influence the perception of a plasticky notebook computer. In many cases, metal components were used in the chassis structure as part of the investment in the system. The need to understand this problem was universal for both Business and Consumer products, since a poorly-perceived chassis will impact product satisfaction and future purchases. Many theories were postulated about the cause of this perception, including variables such as color, industrial design, notebook size, material temperature, material texture, sound of material, and rigidity, all of which were investigated in some capacity in the phases of the study.

\subsection{Method}

Through a combined effort between the Human Factors and Mechanical Engineering teams, a method of identifying the factors and the extent to which they contribute to plasticky perception was developed. One of the difficulties in developing such a strategy was figuring out how to elicit information about specific aspects of the notebook, since many of the factors are interrelated. Some of the factors that were evaluated for their contribution effect were exclusively visually-based, such as color, but others, such as rigidity, might provoke a different response depending on whether the user could see it.

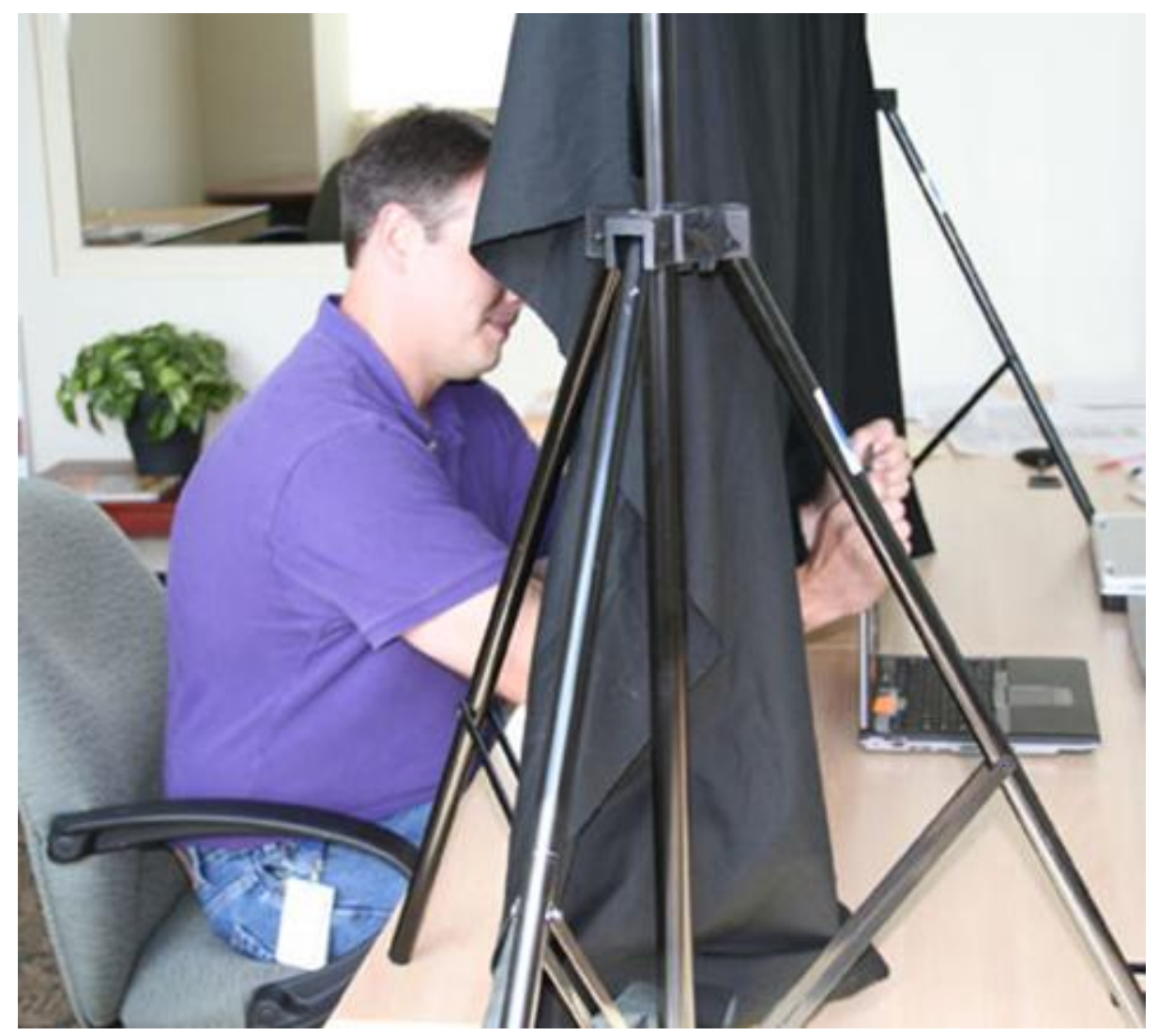

Fig. 4. Eliciting Non-Visual Feedback on System Factors Under Study 
It was determined that the best way to get feedback on the factors was to visibly mask the systems from the user during portions of the study. This was accomplished by using a large, opaque, black cloth that was held up by stands, creating a curtain effect (Figure 4). The test coordinator placed the system being evaluated behind the cloth, allowing the user to reach under the curtain to perform the evaluation (such as touching the notebook palmrest to provide feedback on temperature) without viewing it, thus removing any visual bias. The evaluations were repeated so that the user could see the system. Although somewhat unconventional, this method led to some interesting visual vs. non-visual results, leading us to a better understanding of the impact of each factor. Users were asked to fill out rating scales after each test condition, and the order of the conditions were varied so that the users did not know if they were providing feedback on the same systems for the visual and non-visual portions.

Another difficulty in understanding the extent to which the studied factors impacted plasticky perception involved determining how to take subjective user feedback and relate it to objective measures so that engineers could build to a specification that solved the plasticky problem. Use of 10-centimeter visual analog rating scales allowed for better understanding of the perceived differences of the factors under study. Using the results, we were able to map perceived differences to physical measures that contributed to that perception. As an example, if users rated the rigidity of a specific part on a series of notebooks, we could then map those perceptions against physical measures of rigidity. This was helpful in determining if a mechanical change (and hence, investment in, for example, material thickness, cost, weight, etc.) was even perceptible by users and secondly, whether that perceived difference had an effect on acceptability or other measure of preference.

In the example in Figure 5, if it is assumed that the ratings shown are averages across a number of study participants, it could be inferred that while users could detect differences in perceived component flexibility between System A, System B, and System C, the mechanical measures of rigidity of System A and System B are not "acceptable enough". In other words, gains in perceived quality between the investments made in the component structure of System B may not be worth it, but it may be worth the additional component structure investment in the solution in System $\mathrm{C}$ in order to achieve a perception of high quality.

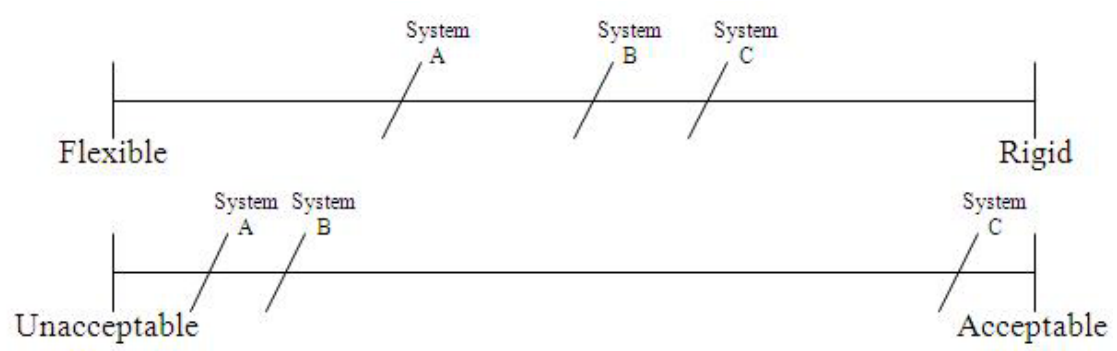

Fig. 5. Example Use of Visual Analog Scales to Determine Extent of Factor Impact 


\subsection{Results}

Once the subjective measures had been gathered, they needed to be mapped to objective data which allows engineers to better evaluate the impact of the differences in user-generated data. Using the same example as depicted in Figure 5, each system's rigidity could be measured at a repeatable, specific point on the system in terms of some unit, such as millimeters of flexion (see Figure 6). Those values could be incorporated into a mechanical specification in such a way that the products that use the specification can be built toward a set value. An example specification statement might be: "Notebook palmrest deflection should not exceed X $-0.6[\mathrm{~mm}]$ of deflection in the z-axis." When this type of approach of marrying subjective data to objective data is applied across multiple attributes and factors, the specifications that can be developed become more robust because they are based on user-generated data. The end goal of using the results to drive improved solutions into the products and reducing the amount of "plasticky" comments was ultimately achieved.



Fig. 6. Example Objective Measures of Rigidity that Can Be Mapped to Subjective Measures

\section{Conclusion}

Oftentimes in the computer industry, human factors engineers are presented with design challenges or questions that cannot be answered with traditional usability testing but require non-traditional methods. These non-traditional methods require creative thought to pinpoint how to best solve and answer the challenge at hand. Just as important as developing the method, the human factors engineer must present the results in engineering terms by providing clear direction as to how a product should be built to meet and exceed customers' expectations rather than simply hand Likert scale ratings to an engineering organization and tell them to "make it better" because it didn't rate well. Additionally, if engineering resources are tight, schedule impact for shipping a product is in jeopardy, or the recommended solution (e.g. a change in material) increases the bill-of-material cost, an executive escalation may occur to move forward with the product design change. In this case, the results must be presentable in a highly visual and succinct way to gain favor with the time-starved executive. Both case studies presented in this paper were embraced by engineers and executives alike primarily due to the manner in which the studies were run and the data presented. While there is obviously no repeatable recipe to follow for a method that is non-traditional, creative forethought on how to design an experiment that targets the question and present the resulting data in a visually engaging and succinct fashion can be the main motivating factor for realizing the implementation of the product design improvement and the resulting positive customer experience. 